\title{
Nivel de funcionalidad en actividades básicas e instrumentales de la vida diaria del adulto mayor
}

\author{
Yessica Chumpitaz Chávez ${ }^{1, a}$, Carlos Moreno Arteaga ${ }^{2, b}$
}

\section{RESUMEN}

La población en general tiene tendencia al envejecimiento, por lo que el Perú debe prepararse con políticas públicas que permitan a este segmento gozar de una vejez digna y con las debidas atenciones. Objetivos: determinar el nivel de funcionalidad en actividades básicas e instrumentales de la vida diaria del adulto mayor del Club CORESAMI en el año 2013. Material y métodos: estudio de tipo cuantitativo, descriptivo, de nivel aplicativo y de corte transversal. La población estuvo conformada por 35 adultos mayores, que concurrían al club antes mencionado, perteneciente a la Jurisdicción del Centro de Salud de San Juan de Miraflores. La técnica que se utilizó fue la encuesta y como instrumento un cuestionario. Resultados: del 100\% (35), un 49\% (17) tiene un nivel medio, un 34\% (12), un nivel alto, y un 17\% (6) un nivel bajo de funcionalidad para realizar actividades básicas e instrumentales de la vida diaria, prevaleciendo las primeras sobre los segundas, para las cuales requieren más apoyo. Conclusiones: se recomienda realizar estudios descriptivos en los diferentes sectores y establecimientos de salud ya que tienen distintas realidades, así mismo programas de intervención dirigidos a estos adultos mayores de carácter inter y multidisciplinario que incluyan a la familia y comunidad.

PALABRAS CLAVE: adulto mayor, funcionalidad, actividades básicas de la vida diaria, actividades instrumentales de la vida diaria.

\section{Level of functionality in basic and instrumental daily life activities of elderly adult}

\section{SUMMARY}

The tendency of the general population is aging, so that Peru should be prepared with public policies that this segment are allowed to enjoy a decent and fairly old-age care. Objectives: to determine the level of functionality in basic and instrumental daily life activities of older adult in CORESAMI Club in 2013. Material and Methods: quantitative and descriptive level application and cross-section study. The population is composed of 35 older adults who were attended in the aforementioned Club, belonging to the jurisdiction of the Health Center of San Juan de Miraflores. The technique used was the survey and a questionnaire as an instrument. Results: of 100\% (35), 49\% (17) having an average level , 34\% (12), a high level, and 17\% (6) a low level of functionality to perform basic and instrumental activities of daily living. Prevailing the first on seconds, for which they require more support. Conclusions: descriptive studies are recommended in different sectors and health facilities as they have different realities, also intervention programs targeting these elderly people, inter and multidisciplinary involving the family and community.

KEYWORDS: el derly adult, functionality, basic activities of daily life, instrumental activities of daily life.

Instituto Nacional Materno Perinatal.Lima, Perú.

Sociedad Científica de San Fernando, Universidad Nacional Mayor de San Marcos. Lima, Perú.

Licenciada en enfermería; ${ }^{\mathrm{b}}$ Estudiante de Medicina. 


\section{INTRODUCCIÓN}

El envejecimiento es un proceso natural, progresivo, de transiciones y cambios psicológicos, sociales y biológicos que aparecen a través del tiempo (1), y el término anciano $o$ adulto mayor es definido por las Naciones Unidas como toda persona mayor de 65 años (países desarrollados) y de 60 años (países en vías de desarrollo). De acuerdo a la Organización Mundial de la Salud (OMS) las personas de 60 a 74 años son consideradas de edad avanzada, de 75 a 90 años son ancianas y mayores de 90 años son grandes viejos (OMS,2005)(2). El aumento de la población adulta mayor ya no es únicamente un fenómeno predominante de los países desarrollados, donde alcanza el 12 a $15 \%$ de la población general, sino también de los países en vías de desarrollo, como es el caso de América Latina $(3,4)$. En el Perú, trabajos de proyección de crecimiento poblacional muestran un $7,4 \%$ de la población y un crecimiento de dicho grupo a $12 \%$ para el año 2025(4). Este aumento de la población adulta mayor a nivel mundial genera preocupación por sus implicancias económicas, sociales y por las condiciones en que este proceso se viene dando (contexto de pobreza e inequidades, falta de cobertura de la seguridad social y una débil red de apoyo producto de los cambios de estructura en la composición familiar y focalización de programas del estado) $(3,5)$. Este grupo social ejerce una fuerte demanda al sistema de salud, debido al incremento de las enfermedades crónicas, los estados de dependencia que muchas de estas patologías (diabetes mellitus, enfermedad cardiaca, respiratoria, cáncer entre otras) y la propia edad terminan por generar (3), situación que produce en el adulto mayor una limitación para satisfacer por sí mismo sus necesidades básicas e instrumentales como lo son la alimentación, evacuación, reposo, sueño, movilidad, hidratación, protección, higiene, confort, entre otras actividades (6).

Se entiende por funcionalidad, a la capacidad del sujeto para realizar sus actividades diarias (4) y funcionalidad geriátrica como la capacidad de un adulto mayor para realizar de manera independiente o autónoma las actividades básicas de la vida diaria o cotidiana (ABVD) e actividades instrumentales de la vida diaria (AIVD) como alimentación, continencia, transferencia, uso del sanitario, vestido, baño y cocinar, realizar las compras, labores domésticas: limpieza de la casa, utilización del teléfono, lavado de la ropa, viajar, tomar medicamentos, administración de los gastos personales, respectivamente $(7,8)$. La presencia del compromiso funcional en el adulto mayor de 65 años puede estar presente en el 5\% y en mayores de 80 años hasta en $50 \%$ o más. La alteración funcional puede ser un "marcador" evidente del efecto de una enfermedad sistémica en el paciente permitiendo de esta manera su reconocimiento, establecer un diagnóstico, pronóstico, una intervención y tipo de cuidado (9). También un indicador de severidad de enfermedad debido a que mide la capacidad de independencia, la cual usualmente se pierde fácilmente con las enfermedades o problemas comunes de este grupo etareo (4). Actualmente, la valoración del estado de salud y calidad de vida del adulto mayor se mira más por la capacidad funcional que por la prevalencia de enfermedades (9). La OMS estableció desde 1999 que la capacidad funcional es el mejor indicador de salud y calidad de vida; por lo tanto, si existe un buen nivel o estado de salud, habrá una buena capacidad funcional y, por consiguiente, calidad de vida (3). A nivel internacional se viene realizando la evaluación geriátrica integral $(3,7)$, y en el Perú, el sistema de evaluación integral geriátrica ha empezado a utilizarse en los pacientes ambulatorios y hospitalizados en Lima y se ha obtenido datos importantes relacionados a la frecuencia de dependencia funcional, deterioro cognitivo, depresión, síndromes geriátricos y otros aspectos relacionados $(4,5)$.

Por lo tanto, la funcionalidad ubicada como parte de la valoración geriátrica y gerontológica resulta importante para el equipo multi e interdisciplinario y para el personal de enfermería que brinda atención a los adultos mayores. Sin embargo, poco se conoce sobre las características de la población adulta mayor en la comunidad y menos aún de aquella que habita en distritos y pueblos fuera de la ciudad de Lima.

Es por ello que el presente trabajo tuvo como objetivo conocer el nivel de funcionalidad en actividades básicas e instrumentales de la vida diaria del adulto mayor del club Conjunto Residencial de San Juan de Miraflores (CORESAMI) en el 2013, con el propósito de que los resultados de esta investigación permitirán al personal de Enfermería y a los otros profesionales de la Salud, tener información para poder elaborar un plan de actividades que fomenten la capacidad funcional de este sector poblacional para mejorar su calidad de vida.

\section{MATERIAL Y MÉTODOS}

El estudio tuvo un enfoque cuantitativo, de tipo descriptivo y corte transversal. Se realizó en el Centro de Salud de primer nivel de atención San Juan de Miraflores perteneciente a la dirección de Salud II Lima Sur, red de servicios San Juan de Miraflores - Villa María del Triunfo, Micro red Trébol Azul ubicada en el pasaje San Juan S/n 
zona A. La población estuvo conformada por los adultos mayores del club Conjunto Residencial de San Juan de Miraflores (CORESAMI) que asistieron al Centro de Salud San Juan de Miraflores, quienes fueron sometidos a los siguientes criterios de selección.

\section{Criterio de inclusión:}

- Tener una edad de 60 años a más.

- Conservar sus facultades mentales y sensorio motrices y valerse por sus propios medios.

- Que acepte participar en el estudio de forma voluntaria.

- Que acuda al Centro de Salud y pertenezca a su jurisdicción.

\section{Criterios de exclusión:}

- Tener edad menor a 60 años.

- Tener enfermedad crónica e invalidante.

- No desee participar en el estudio.

En dicho estudio, la técnica que se utilizó fue la encuesta muestral y como instrumentos fueron los test denominados: índice de Katz para evaluar las actividades básicas de la vida diaria (ABVD) y la escala de Lawton para evaluar las actividades instrumentales de la vida diaria (AIVD). Ambos fueron incluidos en un solo instrumento, el cual constó de tres partes: la primera introductoria; la segunda, de datos generales del informante y la tercera, conformada por los datos específicos de acuerdo a las dimensiones e indicadores del estudio. Fueron 31 ítems de los cuales 8 correspondieron a las ABVD y 23 a las AIVD, asignándoseles puntuación de 0 (si requería de alguna ayuda para realizar una actividad sea básica o instrumental) y 1 (si no requería de ninguna ayuda para realizar sus actividades) dependiendo de cada enunciado. El instrumento, que tuvo como objetivo determinar el nivel de funcionalidad del adulto mayor, fue sometido a validez de contenido y constructo mediante Juicios de Expertos, el cual estuvo conformado por 8 profesionales del campo de la salud con experiencia en salud: del adulto y anciano, investigación y/o afines, 3 enfermeras, 3 docentes de investigación, 1 ingeniero estadístico y 1 médico geriatra. Luego se comprobó su validez y confiabilidad estadísticamente a través del test $\mathrm{R}$ de Pearson y Kuder-Richardson. Posterior a estos pasos, se procedió a categorizar los niveles de funcionalidad y los intervalos, considerando su media, así resultó que:

$$
\begin{array}{lc}
\text { alta } & >=28 \\
\text { medio } & 21-27 \\
\text { bajo } & <=20
\end{array}
$$

Para la recolección de datos se realizaron los trámites administrativos correspondientes y coordinaciones con el Centro de Salud San Juan de Miraflores, con la enfermera jefa del establecimiento de salud y con el personal de salud encargado de los servicios a los que acude el adulto mayor. Previo consentimiento informado, se les entregó el cuestionario, al cual respondieron a las preguntas cerradas marcando con un aspa y completando las preguntas abiertas. Posterior a la recolección, los resultados fueron presentados en tablas y/o gráficos estadísticos para su análisis e interpretación.

Luego de la recolección de datos, estos fueron codificados y procesados mediante el uso del programa Excel y del programa estadístico SPSS 15.0, previa elaboración de la tabla de códigos y la tabla matriz.

El estudio no involucró riesgos para la salud e integridad de los participantes y se mantuvo en todo momento la anonimidad y confidencialidad de los participantes. Se entregó el consentimiento informado, previa información sobre los objetivos y propósitos del estudio y a través de la firma de dicho documento se confirmó la decisión de participar en la investigación, la cual fue respetada por el investigador.

\section{RESULTADOS}

La población sujeto de estudio quedó conformada por 35 adultos mayores, cuyas características demográficas fueron que el $89 \%$ (31 adultos) tenían una edad igual o mayor a 65 años. El 97\% (34 adultos) eran de sexo femenino. En cuanto al grado de instrucción, 31\% (11 adultos), estudiaron primaria completa, el 17\% (6 adultos), estudiaron secundaria completa, otro $17 \%$ ( 6 adultos), tuvieron estudios superiores universitarios completos, el $9 \%$ (3 adultos) tuvieron primaria incompleta, el 6\% (2 adultos) tuvieron estudios técnicos superiores completos mientras que otro $6 \%$ ( 2 adultos), tuvieron estudios superiores universitarios incompletos. Referente a la ocupación, el $71 \%$ (25 adultos) era ama de casa, el 20\% (7 adultos) era jubilada, y el 9\% (3 adultos) cesantes.

En relación al lugar de procedencia, el 66\% (23 adultos) proceden de Lima, y el otro 34\% (12 adultos) son procedentes de provincia. Según la presencia de enfermedades, el 52\% (18 adultos) no padecía ninguna enfermedad.

En cuanto al nivel de funcionalidad en las actividades básicas e instrumentales de la vida diaria de los adultos 
Tabla 1. Nivel de funcionalidad en las actividades básicas e instrumentales de la vida diaria de los adultos mayores del club CORESAMI Lima - Perú 2013

\begin{tabular}{ccc}
\hline $\begin{array}{c}\text { Nivel de funcionalidad en actividades } \\
\text { básicas e instrumentales de la vida diaria }\end{array}$ & N & Adultos mayores \\
\hline Alto & 12 & 34 \\
Medio & 17 & 49 \\
Bajo & 6 & 17 \\
TOTAL & 35 & 100 \\
\hline
\end{tabular}

Tabla 2. Nivel de funcionalidad en las actividades básicas de la vida diaria de los adultos mayores del club CORESAMI Lima - Perú2013

\begin{tabular}{ccc}
\hline $\begin{array}{c}\text { Nivel de funcionalidad en las Actividades } \\
\text { Básicas de la Vida Diaria }\end{array}$ & $\mathbf{N}^{\mathbf{0}}$ & $\mathbf{\%}$ \\
\hline Alto & 34 & 97 \\
Medio & 0 & 0 \\
Bajo & 1 & 3 \\
TOTAL & 35 & 100 \\
\hline
\end{tabular}

Tabla 3. Nivel de funcionalidad en las actividades instrumentales de la vida diaria de los adultos mayores del club CORESAMI Lima - Perú2013

\begin{tabular}{ccc}
\hline Nivel de funcionalidad en las Actividades & \multicolumn{2}{c}{ Adultos Mayores } \\
Instrumentales de la Vida Diaria & $\mathbf{N}^{\mathbf{0}}$ & $\mathbf{\%}$ \\
\hline Alto & 26 & 74 \\
Medio & 8 & 23 \\
Bajo & 1 & 3 \\
TOTAL & 35 & 100 \\
\hline
\end{tabular}

mayores, se obtuvo que del $100 \%$ (35 adultos) de adultos mayores encuestados, el 34\% (12 adultos) tienen un nivel alto, el $49 \%$ (17 adultos) tienen un nivel medio y el 17\% (6 adultos) tienen un nivel bajo (tabla 1$)$.

En cuanto al nivel de funcionalidad para realizar las actividades básicas de la vida diaria de los adultos mayores, se obtuvo que del $100 \%$ de adultos mayores encuestados (35 adultos), el 97\% (34 adultos) tienen un nivel alto, mientras que el 3\% (1 adulto) restante tiene nivel bajo (tabla 2).

En cuanto al nivel de funcionalidad en las actividades instrumentales de la vida diaria de los adultos mayores, se obtuvo que del $100 \%$ de adultos mayores encuestados (35 adultos), el 74\% (26 adultos) tienen nivel alto, el 23\% (8 adultos) tienen un nivel medio y el 3\% (1 adulto) tiene un nivel bajo (tabla 3 ).

\section{DISCUSIÓN}

El envejecimiento de la población es uno de los fenómenos demográficos más importantes en el mundo. En el Perú, la población de personas adultas mayores con más de 60 años viene incrementándose paulatinamente. Según el INEI los departamentos en los que reside el mayor número de personas adultas mayores de 60 años a más son Arequipa, Moquegua, Lima y la Provincia Constitucional del Callao. En tanto que Madre de Dios es el departamento con menor número de población adulta mayor con $4,6 \%$ (10). El progresivo envejecimiento de la población peruana es relativamente reciente, destinado a invertir la pirámide poblacional, y viene dando lugar a una serie de modificaciones sociales, económicas y culturales, que terminan afectando las condiciones y calidad de vida de las personas adultas mayores. Asimismo, de acuerdo con los resultados de la Encuesta Nacional de Hogares de julio 
a setiembre del 2013, el 79,3\% de las mujeres de 60 y más años de edad padecen algún problema de salud crónico (artritis, hipertensión, asma, reumatismo o diabetes, entre otros), mientras que, en los hombres es el $67,4 \%$, es decir, menor en 11,9 puntos porcentuales que las mujeres, por el contrario, los hombres con problemas de salud no crónico representan el 18,0\% y las mujeres el 12,0\% (10).

Según la Organización Panamericana de la Salud (OPS), un adulto mayor posee un alto nivel de funcionalidad cuando es capaz de enfrentar el proceso de cambio en la vejez con un nivel adecuado de adaptabilidad y satisfacción personal. Por ende, la funcionalidad es la capacidad del sujeto para realizar las actividades de la vida diaria (AVD). Esta capacidad declina gradualmente y difiere de individuo a individuo, siendo más notoria en mayores de 65 años. La presencia del compromiso funcional en la persona mayor de 65 años puede estar presente en el $5 \%$ y en mayores de 80 años hasta en $50 \%$ o más, ello depende del estado de salud en que se encuentre el adulto mayor y del impacto de las enfermedades que pudiera tener, sean o no invalidantes y que puedan causarles una limitación funcional en las actividades básicas y/o instrumentales de la vida diaria. Cabe mencionar que la disminución de la funcionalidad puede llevar hasta el grado de la discapacidad, la cual trae consigo dependencia en familiares o cuidadores, lo que genera un impacto, social y económico, importante en la calidad de vida de la persona (8). Lo que coincide con lo informado por Sanhueza Parra y col., quienes afirman que los cambios naturales y problemas de salud que experimenta el adulto mayor se traducen en la declinación de sus capacidades funcionales, las que alcanzan su mayor expresión en el adulto joven y en la etapa de envejecimiento disminuyen notoriamente (11).

Teniendo en cuenta lo antes expuesto y priorizando la capacidad para desempeñar actividades de la vida diaria ya sean básicas o instrumentales, algunas características demográficas evaluadas en el estudio muestran una fuerte asociación con el estado funcional del adulto mayor. Según los estudios previos, la mayor edad de la persona está asociada con la dependencia funcional, tanto para realizar ABVD como AIVD. Esto respalda la afirmación de Guadalupe Dorantes-Mendoza y col., en el sentido de que la mayor edad es quizás el factor de riesgo más importante del deterioro del estado funcional (6). En nuestro estudio el 89\% (31 adultos) tienen 65 años o más $y$ el $34 \%$ (12 adultos) tiene nivel de funcionalidad alta para actividades de la vida diaria. Otro factor asociada significativamente con la dependencia funcional es la presencia de enfermedad o comorbilidad, frecuente en adultos mayores. Es así que según Menéndez y col., encontraron una asociación significativa entre enfermedades no transmisibles, especialmente enfermedad cerebrovascular y la dificultad en realizar actividades de la vida diaria, especialmente AIVD (12).

Respecto a los datos obtenidos en la tabla 1del grupo de adultos mayores evaluados, el $49 \%$ presenta un nivel medio de funcionalidad para realizar actividades de la vida diaria, lo cual quiere decir que el adulto mayor que casi el 50\% de ellos, requieren algún tipo de apoyo de su entorno (familiar, institucional, entre otros), para realizar ciertas actividades de su vida cotidiana; el 34\% de ellos, presentan un alto nivel de funcionalidad, lo que refleja que poseen un gran nivel de independencia para realizar actividades de su vida diaria, no requiriendo de ningún tipo de apoyo; y por último, el 17\% de ellos, tienen un bajo nivel de funcionalidad, quienes tienen cierta limitación para realizar, en su mayoría, actividades de la vida diaria, y que requieren de constante ayuda y supervisión. Está necesidad de apoyo y, por tanto, pérdida de autonomía es una consecuencia del envejecimiento, que sumadas a numerosas alteraciones en la vida del anciano perjudican su calidad de vida, lo que coincide con Lobo A. y col., quienes concluyen en que la pérdida de autonomía puede provocar alteraciones en la calidad de vida y llegar a causar dependencia de otras personas para la realización de las actividades de la vida diaria (13).

De acuerdo a la tabla 2, se obtuvo que 34 de los adultos mayores encuestados (97\%), tienen un alto nivel de funcionalidad, mientras que el 3\% (1 adulto) restante tiene un bajo nivel de funcionalidad. Estos datos coinciden con el estudio de Eduardo Fócil-Némiga y col.,(9). Gloria Villareal Amarís y col.,(3) y Susana Soberanes Fernández y col.,(7); los primeros encontraron que la funcionalidad para $\mathrm{ABVD}$, en un estudio que evaluó a 114 adultos mayores en el poblado C-29, Cárdenas, Tabasco, México, fue normal ( $85 \%$ ); mientras que los segundos, un estudio que evaluó a 275 adultos mayores en dos comunas de Sincelejo, Colombia, encontraron que la mayoría de los adultos son independientes $(73,9 \%)$ para realizar sus ABVD sin depender de alguien; y los últimos, un estudio que evaluó a 225 adultos mayores en una clínica en México, encontraron que el nivel de funcionalidad es normal en $71,6 \%$. Los resultados no coinciden con los estudios de Varela Pinedo Luis y col., quienes encontraron que el $46,5 \%$ tenían algún grado de dependencia para desarrollar sus ABVD (4). 
En relación a la tabla 3, el 74\% de los adultos mayores, presentaron un alto nivel de funcionalidad para realizar actividades instrumentales de la vida diaria. El 23\% de ellos, presenta un nivel medio de funcionalidad, mientras que el $3 \%$ evidencia mayor dependencia. Cifras que no coinciden con los hallazgos de Eduardo FócilNémiga y col., (9) y Gloria Villareal Amarís (3), quienes reportaron que el $66 \%$ con nivel de dependencia funcional instrumental y entre $62,8 \%-47,7 \%$ de dependencia funcional para ciertas AIVD; estas diferencias puede atribuirse quizás a los tipos de poblaciones estudiadas y las actividades que realizan, pues mientras que Eduardo Fócil estudio en ambientes rurales, nuestro estudio se hizo en ambientes urbano y en los estudios de Villareal G., evalúa actividades instrumentales más demandantes. Así mismo nuestro estudio coincide con Susana Soberanes Fernández y cole., quienes encontraron que el $68,4 \%$ de adultos mayores tienen independencia para realizar sus actividades, sin la necesidad de apoyo alguno (7).

Por último, una de las limitaciones es que la presente investigación solo es aplicable a la población estudiada, por ende no se puede generalizar. Entre las recomendaciones se sugiere hacer más estudios de tipo descriptivo sobre el tema para una mejor comparación de los casos en las distintas realidades y/o sectores del país. Asimismo se deben efectuar investigaciones cualitativas que permitan profundizar en los diferentes aspectos que competen a la funcionalidad del adulto mayor y a su vez diseñar programas de intervención que abarque actividades recreativas, sociales y de salud, dirigidos a adultos mayores, involucrandoa la familia y con carácter multidisciplinario.

\section{REFERENCIAS BIBLIOGRAFIAS}

1. Zamora ZEH. Estudio exploratorio sobre el proyecto de vida en el adulto mayor. Psicol Salud. 2014; 16(1):103-10.

2. Mayores de Hoy. El adulto mayor en el Mundo Parte 1. Ciudada de Mexico: Blog de Annika Maya Rivero; 2012 (Citado el 29 de Mayo del 2016) Disponible en: https:// mayoresdehoy.com/2012/11/07/el-adulto-mayor-en-elmundo-parte-1/

3. Villarreal G, Month E. Social and family, health care and functional conditions of elderly people over 65 year old in two neighborhoods of Sincelejo (Colombia). Rev Salud Uninorte. 2012;28(1):75-87.

4. Varela L, Chávez H, Galvez M, Mendez F. Funcionalidad en el adulto mayor previa a su hospitalización a nivel nacional. Rev Medica Hered. 2005;16(3):165-71.

5. Ruiz L, Campos M, Peña N. Situación sociofamiliar, valoración funcional y enfermedades prevalentes del adulto mayor que acude a establecimientos del primer nivel de atención, Callao 2006. Rev Peru Med Exp Salud Publica. 2008; 25(4):374-9.

6. Dorantes-Mendoza G, Ávila-Funes JA, Mejía-Arango S, Gutiérrez-Robledo LM. Factors associated with functional dependence in older adults: a secondary analysis of the National Study on Health and Aging, Mexico, 2001. Rev Panam Salud Pública. 2007; 22(1):1-11.

7. Fernández SS, González Pedraza Avilés A, Castillo Y del CM. Funcionalidad en adultos mayores y su calidad de vida. Rev Espec Méd-Quirúrgicas. 2009;14(4):161-75.

8. Organización Panamericana de la Salud. Evaluación Funcional del Adulto Mayor. Washington: Organización Panamericana de la Salud (Citado el 29 de Mayo del 2016) Disponible en: http://www.sld.cu/galerias/pdf/sitios/ gericuba/modulo3.pdf

9. Fócil-Némiga E, Zavala-González MA. Funcionalidad para actividades de la vida diaria en adultos mayores rurales de Cárdenas, Tabasco, México. Rev Fac Salud - RFS. 2015; 6(2):12-9.

10. Instituto Nacional de Estadística e Informática. Perú: Situacion de la salud de la población Adulto Mayor, 2012. Lima: Instituto Nacional de Estadística e Informática ;2013 (Citado el 29 de Mayo del 2016) Disponible en: https://www. inei.gob.pe/media/MenuRecursivo/publicaciones_digitales/ Est/Lib1146/libro.pdf

11. Sanhueza M, Castro M, Escobar M. Adultos mayores funcionales: Un nuevo concepto en salud. Cienc Enferm. 2005;11(2):17-21.

12. Menéndez J, Guevara A, Arcia N, et al. Chronic diseases and functional limitation in older adults: a comparative study in seven cities of Latin America and the Caribbean. Rev Panam Salud Pública. 2005;17(5-6):353-61.

13. Lobo A, Paula- Santos M, Carvalho J. Anciano institucionalizado: calidad de vida y funcionalidad. Rev Esp Geriatría Gerontol. 2007;42(S1):22-6.

\section{Correspondencia:}

Yessica Chumpitaz Chávez

Correo electronic: yessika_239@hotmail.com

Fecha de Recepción: 31 de mayo del 2016.

Fecha de aceptación: 20 de junio del 2016. 
